\title{
Endocytosis of fluorescent microspheres by human oesophageal epithelial cells: comparison between normal and inflamed tissue
}

\author{
D Hopwood, E M Spiers, P E Ross, J T Anderson, J B McCullough, F E Murray
}

\begin{abstract}
This paper examines the presence and characteristics of endocytosis by oesophageal epithelial cells. Biopsy specimens from normal and inflamed oesophagus were incubated in organ culture with fluorescent microspheres $(0.1$ and $0.01 \mu \mathrm{m}$ diameter). These markers were taken into early endosomes and the lysosomes of both the smaller differentiating prickle cells and the larger mature squamous cells. Confocal and electron microscopy showed that markers passed to the early endosomes and the lysosomes by endocytosis. The process was energy dependent. Larger, $1 \mu \mathrm{m}$ microspheres adhered to the epithelial cells but were not phagocytosed. Disaggregated cells were analysed by flow cytometry. Microspheres were endocytosed in proportion to the concentration in the culture medium in a dose dependent manner. Cells from inflamed oesophagus were significantly smaller $(p=0.013)$ and took up significantly more microspheres than cells from normal biopsy specimens $(p=0 \cdot 015)$. In conclusion, endocytosis occurs in oesophageal epithelial cells and is increased in inflammation.

(Gut 1995; 37: 598-602)
\end{abstract}

Keywords: endocytosis, oesophagus, oesophagitis.

The metabolic activity of oesophageal epithelium has been little studied. Orlando's group have investigated the ability of whole oesophagus and cultured cells to withstand the effects of acid damage. ${ }^{12}$ We have shown that the oesophageal epithelial cells can secrete and endocytose cationised ferritin ${ }^{3}$ and mucosubstances. ${ }^{4}$ This latter observation is an important constitutive function of almost all eucaryotic cells, whereby extracellular fluid and its solutes are transported into the cell.

This study aimed to evaluate the ability of normal oesophageal cells to ingest fluorescent microspheres, to investigate the intracellular pathway followed by the ingested microspheres, and to enumerate uptake by the different oesophageal cell types. Furthermore, we compared these parameters in normal and inflamed oesophageal tissue.

To this end, we used fluorescein labelled latex spheres, which have a diameter of 0.01 $\mu \mathrm{m}$ and $0 \cdot 1 \mu \mathrm{m}$ and act as fluid phase markers. Oesophageal cells were exposed to the microspheres in organ culture. We have previously established the viability of oesophageal biopsy tissue in organ culture and this approach allowed maximum control of experimental conditions. ${ }^{5}$ The intracellular pathways of ingested microspheres were investigated by confocal and electron microscopy. Disaggregation of cells from the biopsy tissues after organ culture with microspheres allowed analysis by flow cytometry, permitting quantitative investigation of normal and inflamed specimens.

\section{Methods}

Oesophageal pinch biopsy specimens were obtained from patients undergoing upper gastrointestinal endoscopy. Written informed consent had been obtained beforehand. One group of patients had endoscopically and histologically normal oesophageal mucosa $(n=14)$ and the other group had endoscopically and histologically inflamed oesophageal mucosa $(n=6)$. Biopsy specimens were placed in tissue culture medium

\section{ORGAN CULTURE}

This was basically as described by Browning and Trier $^{6}$ and previously used in our laboratory. ${ }^{4}{ }^{5}$ The biopsy specimens were collected in phosphate buffered saline (PBS) on ice in the endoscopy suite and cultured overnight at $37^{\circ}$ in $95 \%$ oxygen and $5 \%$ carbon dioxide in organ culture dishes on a stainless steel grid at the fluid/gas interface.

Specimens were transferred to tissue culture medium used (Ham's F10) containing penicillin $(100 \mathrm{IU} / \mathrm{ml})$ streptomycin $(100 \mu \mathrm{g} / \mathrm{ml})$, and fungizone $(2.5 \mu \mathrm{g} / \mathrm{ml}$ ) (all from Gibco Life Technologies, Paisley). Prepared coated fluorescent microspheres were added (Cambridge BioScience, Cambridge).

FLUORESCENT MICROSPHERES

Carboxylate modified latex microspheres 
with diameters of $0.01,0.1$, and $1.0 \mu \mathrm{m}$ $( \pm 1 \%)$ and labelled internally with yellowgreen fluorescent dye were incubated with $1 \%$ (w/v) albumin for one hour in an ultrasonic bath to reduce aggregation of the larger spheres. The albumin solution containing the microspheres was diluted in Ham's F10 medium to give between $6.5 \times 10^{5}$ and $6.5 \times 10^{12}$ spheres $\mathrm{ml}^{-1}$ for organ culture. The yellow-green dye was activated at $490 \mathrm{~nm}$ appropriate for the argon laser and emission was detected at $515 \mathrm{~nm}$.

\section{DISAGGREGATION}

The biopsy specimens were largely disaggregated by their own proteinase activity after agitation for 30 seconds with a Whorlimix and gentle pipetting through a capillary tube. The disaggregated cells were washed twice with PBS and pelleted by spinning for five minutes at $1000 \mathrm{~g}$. The supernatant washings were decanted and cells were fixed in $0.5 \%$ paraformaldehyde in PBS. The fixed cells were stored for up to three days at $4^{\circ} \mathrm{C}$ in the dark before flow cytometry.

\section{FLOW CYTOMETRY}

The cells were analysed using a Beckton and Dickinson FAC scan. The parameters were measured and analysed by using LYSIS II software.

CONFOCAL MICROSCOPY

Disaggregated cells were examined with a BioRad MRC 600 confocal microscope under an oil immersion lens.

\section{ELECTRON MICROSCOPY}

Tissues or disaggregated cells were fixed in 3\% glutaraldehyde $\mathrm{pH} \quad 7 \cdot 2$ and embedded in Araldite. These sections stained with uranyl nitrate lead citrate were examined at $50 \mathrm{kV}$ in a JEOL 100CX electron microscope. Droplets of microspheres were negatively stained with phosphotungstic acid before observation.

\section{LIGHT MICROSCOPY}

Haematoxylin and eosin paraffin sections of biopsy specimens from sites adjacent to those specimens taken for organ culture were examined histopathologically. Droplets of disaggregated cells were fixed and stained with haematoxylin and eosin as a control for the cellular preparations. Drop preparations of disaggregated cells were also examined by fluorescent microscopy.

\section{Results}

After incubation overnight, the specimens from normal oesophagus maintained a discrete structure and could be easily picked off the wire mesh with forceps. The biopsy specimens from patients with an inflamed oesophagus, however, had a more gelatinous appearance after incubation. They tended to fragment so that they were more difficult to pick up. The oesophageal cells from normal biopsy tissues endocytosed bovine serum albumin coated microspheres with diameters of 0.01 and 0.1 $\mu \mathrm{m}$. The largest fluorescent microsphere available from BioScience $(1 \mu)$ was not endocytosed. Endocytosis of microspheres seemed to depend on both time and temperature.

\section{LIGHT MICROSCOPY}

Drop preparations confirmed the nature of tissues examined and minimal contamination by connective tissue. Haematoxylin and eosin sections of adjacent biopsy specimens confirmed that the oesophageal tissue was histologically normal or inflamed. No candida was seen.

Fluorescence microscopy showed that the fluorescent spheres bound to the surface of the oesophageal epithelial cells with some possible internalisation. The smaller prickle cells seemed to fluoresce more intensely than the larger mature squamous cells.

\section{CONFOCAL MICROSCOPY}

The endocytosis of the fluorescent microspheres was confirmed by confocal microscopy. Fluorescent bodies were seen at the cell periphery corresponding to the early endosomal compartment (Fig 1). There were also larger fluorescent bodies around the nuclei, perinuclear lysosomes. The smaller cells had a greater cytoplasmic fluorescent intensity than the larger cells, in which the fluorescent bodies were more widely dispersed (Fig 2).

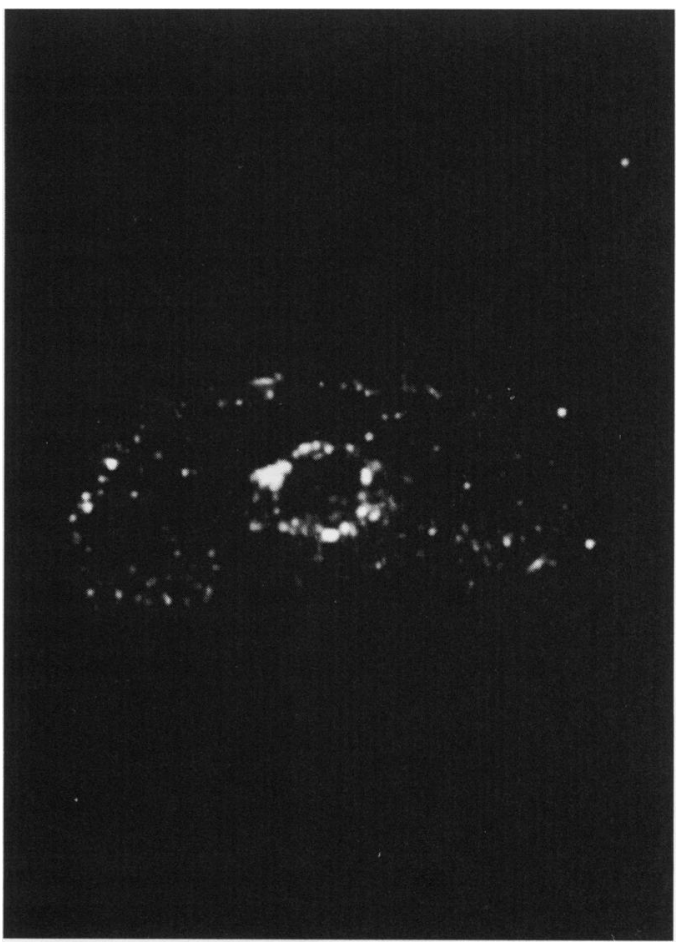

Figure 1: Confocal microscopy. Mature squame showing fluorescent microspheres in the periphery of the cytoplasm (early endosomes). Around the nucleus there are a number of larger fluorescent bodies (lysosomes). 
Figure 2: Confocal microscopy. Prickle cells with an intense zone of cytoplasmic fluorescence surrounding the central nucleus.
Figure 3: Electron micrograph of negatively stained microspheres showing their uniform size (original magnification $\times 20000)$.

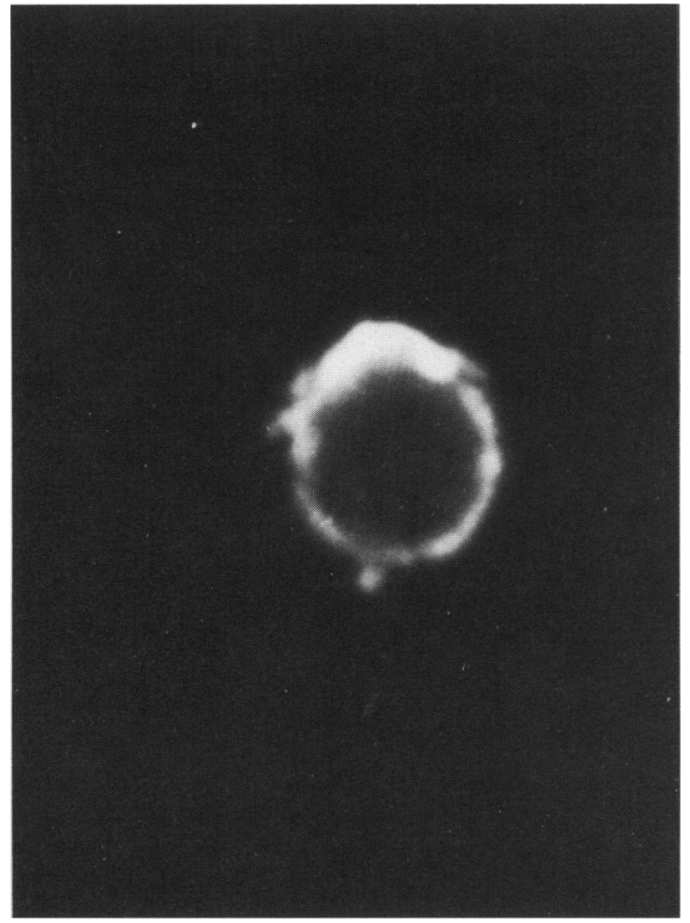

\section{ELECTRON MICROSCOPY}

The microspheres were of a uniform size (Fig 3). They could be seen in early endosomes in smaller numbers (Fig 4). Lysosomes contained larger numbers of microspheres corresponding to the confocal findings (Fig 5).

\section{FLOW CYTOMETRY}

The linear and logarithmic dot plots had two distinct appearances (Fig 6), the latter showing a distinct population of cells. Polymorphonuclear leukocytes were used as size markers $(15 \mu \mathrm{m})$ to exclude debris and indicate the size of the smallest of the oesophageal cells. The size of the individual cells as forward scatter and the mean fluorescence were measured, both in arbitrary units.

There was a direct relationship between the concentration of microspheres in the incubating medium and the mean fluorescence of the oesophageal cells (Fig 7).

Endocytosis of spheres by oesophageal cells from normal and inflamed biopsy specimens was measured. The mean diameter of cells

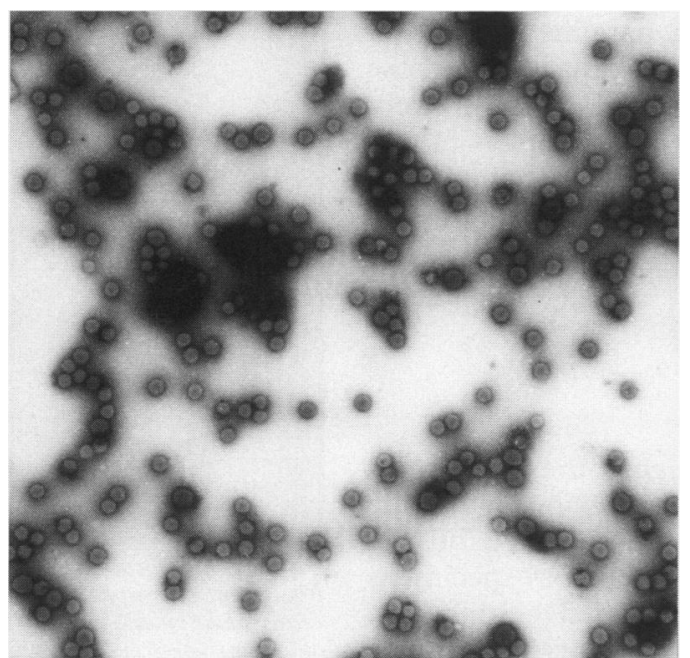

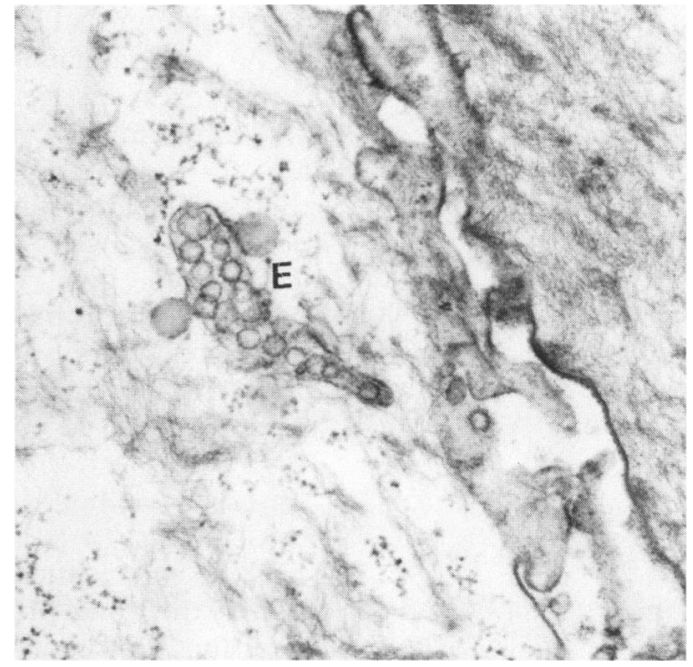

Figure 4: Electron micrograph showing two functional cells. There are a number of microspheres in an early endosome $(E)$ (original magnification $\times 26000$ ).

from the inflamed specimens were significantly smaller than normal. In addition, inflamed cells took up significantly more spheres than the uninflamed cells (Table) (Fig 8). The fluorescence of small cells from normal and inflamed specimens was compared. There was a trend for cells from inflamed biopsy specimens to be more fluorescent but this did not reach statistical significance.

\section{Discussion}

In this study we have shown that oesophageal epithelial cells endocytose fluorescent microspheres of diameter $0 \cdot 01-0 \cdot 1 \mu \mathrm{m}$. Endocytosis was dose dependent and energy dependent. Endocytosis was more marked in smaller epithelial cells than in the large mature squames. Furthermore, inflamed oesophageal epithelium endocytosed to a greater extent than normal oesophagus.

Organ culture is a well established technique that has been used in investigations of various parts of the gastrointestinal tract. ${ }^{6} \mathrm{We}$

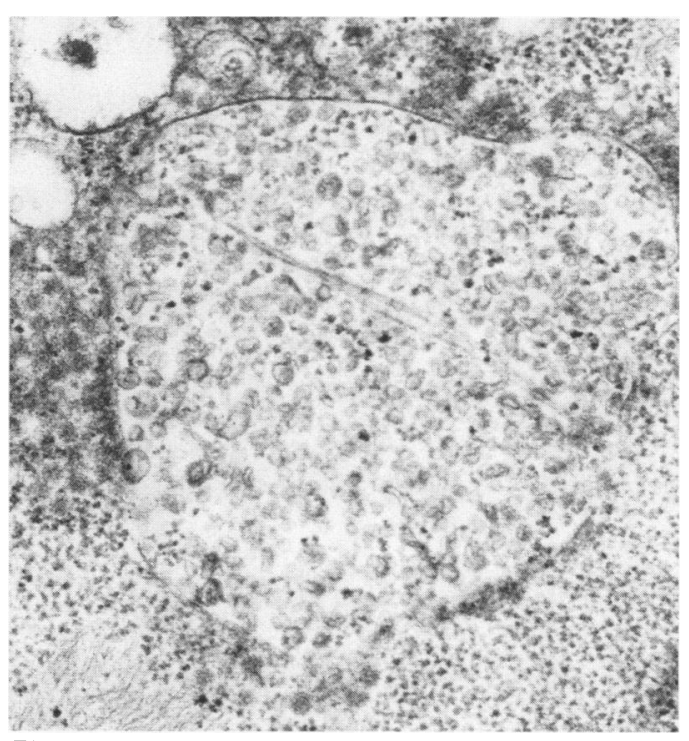

Figure 5: Electron micrograph of a prickle cell showing ysosome which contains large numbers of microspheres (original magnification $\times 32000$ ). 


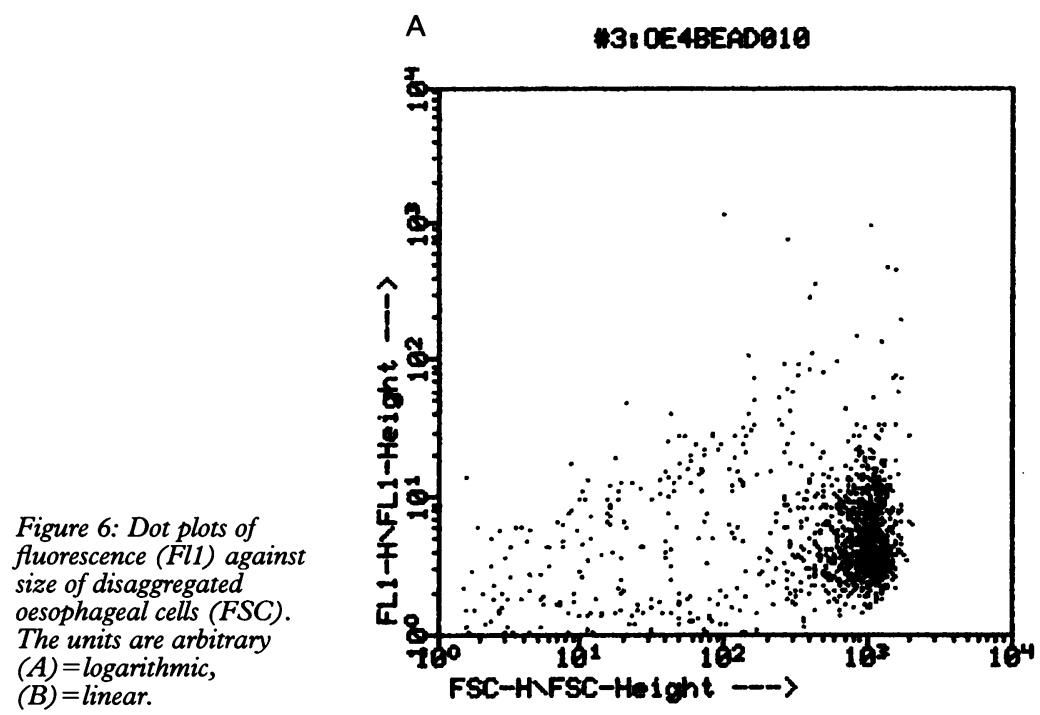

have previously used this technique with endoscopic oesophageal mucosal biopsy specimens. ${ }^{4}$ We found that after 18 hours' organ culture the cells had normal ultrastructural morphology and, functionally, were able to endocytose horseradish peroxidase. ${ }^{5}$ Movement of substances between various intracellular compartments could be manipulated in organ culture.

The oesophageal squamous epithelium is held together by desmosomes, integrins, and E-cadherin and attached to the basement membrane by hemidesmosomes. ${ }^{7}$ A number of techniques have been used to disaggregate oesophageal cells, most utilising a proteinase and some with chelating agents. ${ }^{8}$ The resulting disaggregated cells are $90-95 \%$ epithelial. We have shown that oesophageal epithelial biopsy specimens left overnight in organ culture will largely loosen intercellular adhesion and may be disaggregated by mild physical techniques. ${ }^{9}$ This seems to be due to an endogenous proteinase which can be inhibited by aprotinin. A similar phenomenon has also been shown with skin biopsy tissues. ${ }^{10}$

The advantages of using the organ culture technique are that the microanatomical relations between cells remain intact and no exogenous substances that might change the cell surfaces are introduced. By holding the cells in the form of tissue, however, access to the cells of probes is not uniform and will depend on time.

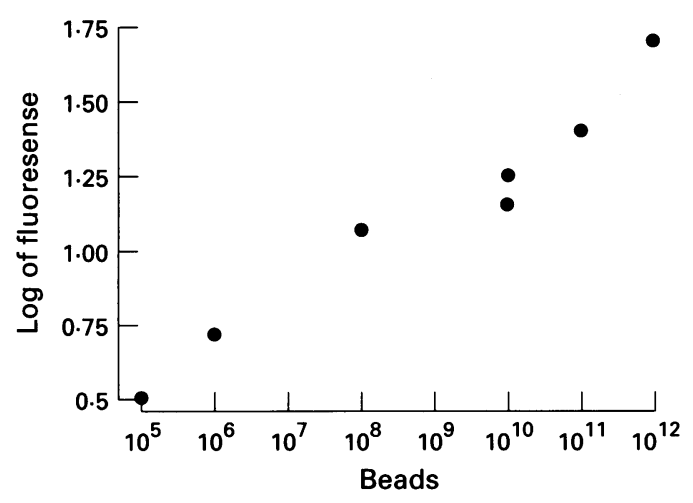

Figure 7: Plot of the log mean fluorescence of oesophageal cells against the numbers of beads per $m l$ in the incubation medium.

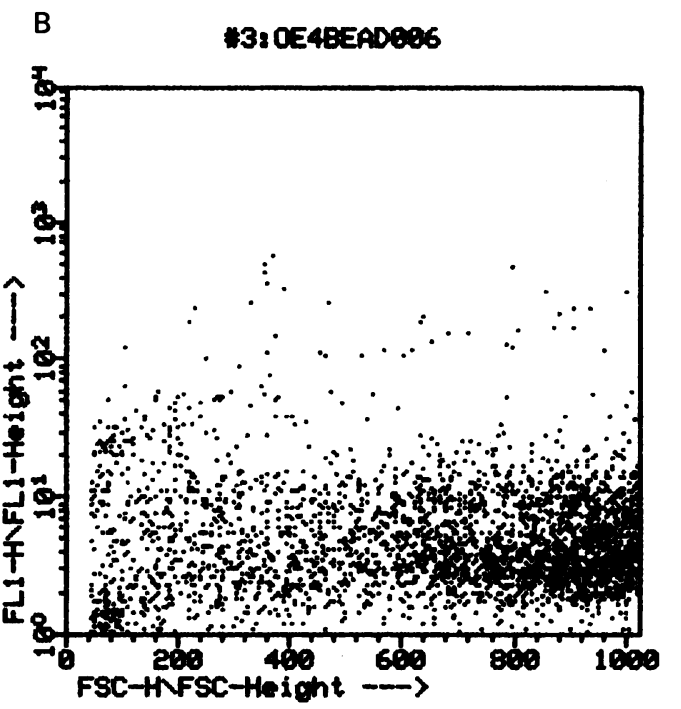

In the present experiments, we utilised microspheres coated by bovine serum albumin to prevent aggregation. They may be coated by other substances. We have previously coated latex microspheres with antibodies to CD15 and shown, by scanning electron microscopy, that the microspheres adhered to the surface membranes of oesophageal squamous cells. ${ }^{11}$ Fluorescent microspheres of three sizes are available $(0 \cdot 01-1 \mu)$. We found that the two smaller ones are markers of the fluid phase and were readily taken into the epithelial cells by endocytosis. Phagocytosis as defined by the size of particle ingested 0.5-1 $\mu \mathrm{m}$ was not observed, although the underlying processes are believed to be the same as endocytosis. ${ }^{12}$ It has previously been shown that keratinocytes can phagocytose thorotrast particles. ${ }^{13}$

We showed that fluorescent microspheres were endocytosed by both the large mature squamous cells and the smaller differentiating prickle cells. In the former, the microspheres were distributed more diffusely in a larger cell volume than in the latter. There was no significant difference in the fluorescence of small cells derived from normal or inflamed oesophageal biopsy specimens, although there was a trend for the latter to be higher. The lack of statistical significance may be a result of the small number of patients in each group. The higher mean fluorescence in the inflamed

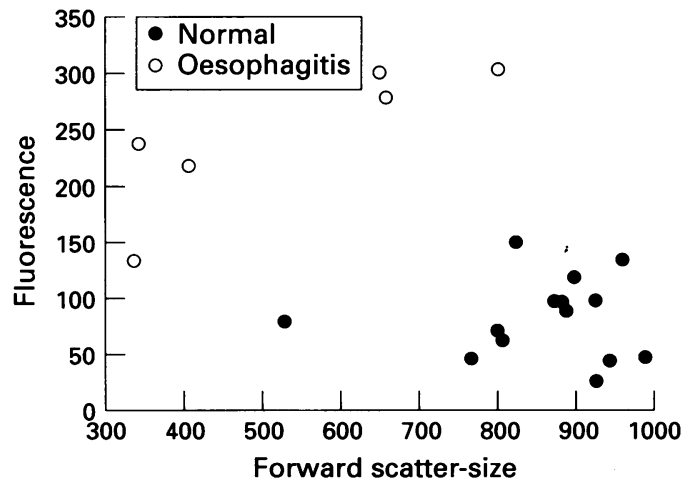

Figure 8: Plot of mean fluorescence against mean size of cells from disaggregated oesophageal biopsy specimens. 
Comparison of size and fluorescence of epithelial cells from normal and inflamed biopsy specimens

\begin{tabular}{lllll}
\hline & $\begin{array}{l}\text { Biopsy } \\
\text { specimen }\end{array}$ & No & Mean (SD) & $p$ \\
\hline Size & Normal & 6 & $835(152)$ & $4.4 \times 10^{-3}$ \\
Fluorescence & Inflamed & 8 & $538(189)$ & \\
& Normal & 6 & $101(66)$ & $3.4 \times 10^{-3}$ \\
& Inflamed & 8 & $256(63)$ & \\
\hline
\end{tabular}

Size and fluorescence are in arbitrary units.

group may be due to the effect of cytokines. We have shown in pilot experiments that epidermal growth factor increases appreciably the uptake of fluorescent microspheres.

The microspheres entered by the early endosomes and were deposited in the perinuclear lysosomes, where, in a single section, 300-400 microsphere profiles could be seen by electron microscopy. When biopsy specimens were incubated for a shorter time, fewer microspheres were taken up.

The labelled epithelial cells were examined by flow cytometry to produce dot plots of cell size (forward scatter) against fluorescence and to calculate their mean values. The linear plots were similar to those we have already described. Logarithmic plots produced a readily discernible population of cells. The oesophageal cells had been measured and were compared against human lymphocytes and polymorphonuclear leukocytes. This allowed debris to be excluded.

Normal oesophageal biopsy specimens were used to investigate the effect of increasing the number of microspheres on the extent of endocytosis. Using constant conditions of incubation, it was possible to show that the more microspheres present in the incubation medium, the greater the uptake. No plateau was reached in the present system. This may represent a diffusion problem associated with the organ culture model. We have previously shown that epidermal growth factor receptor is present, especially on prickle cell membranes. ${ }^{8}$ This suggests that incubation with epidermal growth factor should increase the uptake of microspheres. Other factors such as tumour growth factor- $\alpha$ may produce similar effects.

We also compared the endocytosis of fluorescent microspheres of normal and inflamed oesophageal biopsy specimens. The epithelial cells from the inflamed oesophageal tissue showed a significantly higher mean fluorescence and were significantly smaller than epithelial cells derived from normal specimens. These smaller cells could be seen on the dot plots. More immature cells are also seen histopathologically in oesophagitis.

Endocytosis is a constitutive property of most eukaryotic cells. This allows the internalisation of nutrients and receptor mediated substances. Epidermal keratinocytes have previously been shown to phagocytose thorium dioxide particles. Endocytosis is also a mechanism that may help control the amount of intercellular material. This may play a part in oesophageal barrier function. We found that endocytosis occurred with $0.2 \mu \mathrm{m}$ diameter microspheres. In life, this may represent debris in the intercellular space, and also viruses. Herpes simplex has been reported in a quarter of oesophageal ulcers.

The smaller inflamed cells also showed a greater range of fluorescence than the controls. These findings fit well with histological observations in oesophagitis, where an increase in the basal and parabasal cells can be seen. ${ }^{14}$ The mitotic index has also been shown to increase in oesophagitis using tritiated thymidine and proliferating cell nuclear antigen. ${ }^{15}$ Morphologically, the prickle cells seem more active metabolically than the mature squamous cells.

In conclusion, oesophageal biopsy specimens grown in organ culture can endocytose fluorescent microspheres. The specimens were disaggregated and analysed by flow cytometry. A dose response curve was established for the fluorescence of the cells in relation to the number of microspheres in the incubation medium. Specimens from inflamed oesophagus showed a significant increase in the endocytosis of the microspheres and a significant decrease in the mean size. This technique may prove useful in investigating the tissue response to various treatments for reflux oesophagitis.

An abstract of this paper has already been published: Hopwood D, Spiers E, Ross PE, Anderson JT, McCullough J, Murray FE. Gut 1994; 35 (suppl 5): S14.

1 Tobey NA, Orlando RC. Mechanisms of acid injury to rabbit oesophageal epithelium. Gastroenterology 1991; 101: $1220-8$

2 Tobey NA, Reddy SP, Keku TO, Cragoe EJ, Orlando RC. Studies of pHi in rabbit esophageal basal and squamous epithelial cells in culture. Gastroenterology 1992; 103: 830-9.

3 Hopwood D, Curtis M, Nicholson G, Milne G. The distribution and mobility of surface anionic groups of normal human oesophageal epithelium following interaction with cationized ferritin. Virchows Archiv 1979; B31: 277-88.

4 Hopwood D, Milne G, Jankowski J, Howat K, Johnston D, Wormsley KG. Secretory and absorptive activity of oesophageal epithelium: evidence of circulating mucosubstances. Histochem $¥$ 1994; 26: 41-9.

5 Hopwood D, Milne G, Jankowski J, Howat K, Wormsley KG. Uptake of horseradish peroxidase by human KG. Uptake of horseradish peroxidase by human oesophageal

6 Browning TH, Trier JS. Organ culture of mucosal biopsies of human small intestine. F Clin Invest 1969; 48: 1423-32.

7 Dobson H, Pignatelli M, Hopwood D, D'Arrigo C. Cell adhesion molecules in oesophageal epithelium. Gut 1994; 35: 1343-7.

8 Hopwood D, Jankowski J, Milne G, Wormsley KG. Flow cytometry of oesophageal mucosal biopsies: epidermal growth factor receptor and CD15. F Pathol 1992; 167: $321-6$.

9 Hopwood D, Dobson H, Jankowski J, Howat K, Milne G, Wormsley KG. Mechanisms involved in the release of superficial squames from oesophageal squamous mucosa. Histochem $\mathcal{f}$ 1992; 24: 548 .

10 Lundstrom A, Egelrud T. Cell shedding from human plantar skin in vitro: evidence of its dependence on endogenous proteolysis. F Invest Dermatol 1988; 91: 340-3.

11 Sanders DSA, Kerr MA, Hopwood D, Coghill G, Milne G. Expression of the 3-fucosyl N-acetyllactosamine (CD15) antigen in normal, metaplastic, dysplastic and neoplastic squamous epithelial. F Pathol 1988; 154: 255-62.

12 Silverstein SC, Greenberg S, di Virgilio F, Steinberg TH. Phagocytosis. In: Paul WE, ed. Fundamental immunolog. New York: Raven Press, 1991: 703-20

13 Wolf $\mathrm{K}$, Honigsmann $\mathrm{H}$. Permeability of the epidermis and the phagocytic activity of keratinocytes. Ultrastructural studies with thorotrast as a marker. fournal of Ultrastructural Research 1971; 36: 176-90.

14 Day DW. Infective, physical and chemical oesophagitis. In: Whitehead R, ed. Gastrointestinal and oesophageal pathology. Whitehead R, ed. Gastrointestinal and oesophageal path

15 Jankowski J, Austin W, Howat K, Coghill G, Hopwood D, Dover $R$, et al. Proliferating cell nuclear antigen in Eur $\mathfrak{\exists}$ Gastroenterol Hepatol 1992; 4: 579-84. 